PROCEEDINGS OF THE

AMERICAN MATHEMATICAL SOCIETY

Volume 134, Number 11, November 2006, Pages 3247-3253

S 0002-9939(06)08685-0

Article electronically published on June 6, 2006

\title{
COMPARISON OF POTENTIAL THEORETIC PROPERTIES OF ROUGH DOMAINS
}

\author{
KRZYSZTOF BURDZY AND ZHEN-QING CHEN
}

(Communicated by Michael T. Lacey)

\begin{abstract}
We discuss the relationships between the notions of intrinsic ultracontractivity, the parabolic Harnack principle, compactness of the 1-resolvent of the Neumann Laplacian, and the non-trap property for Euclidean domains with finite Lebesgue measure. In particular, we give an answer to an open problem raised by Davies and Simon in 1984 about the possible relationship between intrinsic ultracontractivity for the Dirichlet Laplacian in a domain $D$ and compactness of the 1-resolvent of the Neumann Laplacian in $D$.
\end{abstract}

\section{INTRODUCTION AND REVIEW OF RESULTS}

In a recent joint paper $[\mathrm{BCM}$ with Don Marshall, we studied a class of domains (called "trap domains") with rough boundaries defined in a potential theoretic way. The main goal of that paper was to give a geometric characterization of trap domains. A number of families of rough domains had been introduced in potential theoretic literature. It is desirable to know which of these families are contained in other families, so that known results can be extended to freshly defined families without duplication of effort. We examined several families of domains that initially seemed to be related to non-trap domains. The results of this paper show that there is no logical relationship between these families, except for some "obvious" cases. In the rest of the introduction we will define four classes of domains and outline their relationships. The next section will present the proofs.

We will now recall the definitions of trap domains, the parabolic Harnack principle, 1-resolvent, and intrinsic ultracontractivity. Throughout this paper, $D$ is a connected open set in $\mathbb{R}^{d}$ with finite Lebesgue measure.

Trap domains. Let $B \subset D$ be a closed ball with non-zero radius and let $G(x, y)$ be the Green function for the domain $D \backslash B$ with the (zero) Neumann boundary conditions on $\partial D$ (in the distributional sense) and (zero) Dirichlet boundary conditions on $\partial B$ (see [BCM] for existence and uniqueness). The domain $D$ is called a trap domain if

$$
\sup _{x \in D \backslash B} \int_{D \backslash B} G(x, y) d y=\infty .
$$

Received by the editors May 17, 2005.

2000 Mathematics Subject Classification. Primary 35P05; Secondary 60J45.

Key words and phrases. Neumann Laplacian, trap domain, intrinsic ultracontractivity, parabolic Harnack principle.

This research was partially supported by NSF grant DMS-0303310.

(C)2006 American Mathematical Society 3247

Reverts to public domain 28 years from publication 
The definition of a trap domain does not depend on the choice of the ball $B$ (see Lemma 3.3 of $[\mathrm{BCM}])$. Probabilistically, (1.1) is equivalent to

$$
\sup _{x \in D \backslash B} \mathbb{E}_{x} \sigma_{B}=\infty
$$

where $\sigma_{B}$ is the first hitting time of $B$ by the normally reflected Brownian motion (RBM in abbreviation) on $D$. See $B \overline{B C M}$ for an account of the construction of RBM on non-smooth domains and its properties. When (1.1) holds, the RBM on $D$ will spend a large amount of time near the boundary of $D$; this is why we call such a domain a "trap domain". A domain $D$ that is not a trap domain will be called a non-trap domain. The notion of a non-trap domain is closely related to the uniform ergodicity of reflected Brownian motion on $D$ (see Proposition 1.2 of [BCM] $)$.

Parabolic Harnack principle. Let $p(t, x, y)$ be the heat kernel on $D$ with zero Neumann boundary condition, or equivalently, the transition density function with respect to the Lebesgue measure on $D$ of the RBM on $D$ (see $[\mathrm{BCM}$ for the definition and existence). We will say that the parabolic Harnack principle (PHP) holds in $D$ if for some $t_{0}>0$ and $c_{1}=c_{1}\left(D, t_{0}\right)<\infty$,

$$
p(t, x, y) \leq c_{1} p(t, v, z) \quad \text { for all } t \geq t_{0} \text { and } v, x, y, z \in D .
$$

Resolvent. Let $P_{t}$ be defined for $t \geq 0$ by $P_{t} f(x)=\int_{D} p(t, x, y) f(y) d y$. Then the 1-resolvent $R_{1}$ is given by $R_{1} f(x)=\int_{0}^{\infty} e^{-t} P_{2 t} f(x) d t$. It is known (see, e.g., Lemma 3.1 of $[\mathrm{BCM}]$ ) that the 1-resolvent $R_{1}$ of the Neumann Laplacian in $D$ is a compact operator in $L^{2}(D, d x)$ if and only if the Neumann Laplacian in $D$ has discrete spectrum.

Intrinsic ultracontractivity. Let $T_{t}$ be the semigroup for the Dirichlet Laplacian in $D$ conditioned by the first Dirichlet eigenfunction through Doob's $h$-transform. That is, with $\varphi_{1}>0$ denoting the positive eigenfunction for the Dirichlet Laplacian in $D$ corresponding to the first eigenvalue $\lambda_{1}<0$, define

$$
T_{t} f(x)=e^{-\lambda t} P_{t}^{D}(\varphi f)(x) / \varphi(x) .
$$

Here $P_{t}^{D}$ is the semigroup for the Dirichlet Laplacian in $D$. We say that $D$ is intrinsically ultracontractive (IU) if $T_{t}$ maps $L^{2}\left(D, \varphi(x)^{2} d x\right)$ into $L^{\infty}(D, d x)$ for every $t>0$ (see Davies and Simon [DS1]).

We will now discuss the relationships between various properties of domains defined above.

It is well known that, for a domain with finite volume, a uniform bound for the heat kernel, such as (1.2), implies that the 1-resolvent of the Neumann Laplacian is compact and therefore the Laplacian in $D$ with the Neumann boundary conditions has a discrete spectrum; see [BB, p. 6] for a typical application.

The following was established in $[\mathrm{BCM}]$ as Proposition 1.4 (ii)-(iii).

Proposition 1.1. (a) If the parabolic Harnack principle holds in $D$, then $D$ is non-trap.

(b) There exists a non-trap domain where the 1-resolvent of the Neumann Laplacian is not compact and, therefore, the parabolic Harnack principle does not hold.

We will show in Theorem 2.1 below that there is a trap domain $D$ where the 1-resolvent of the Neumann Laplacian on $D$ is compact. 


\section{Parabolic Harnack Principle}

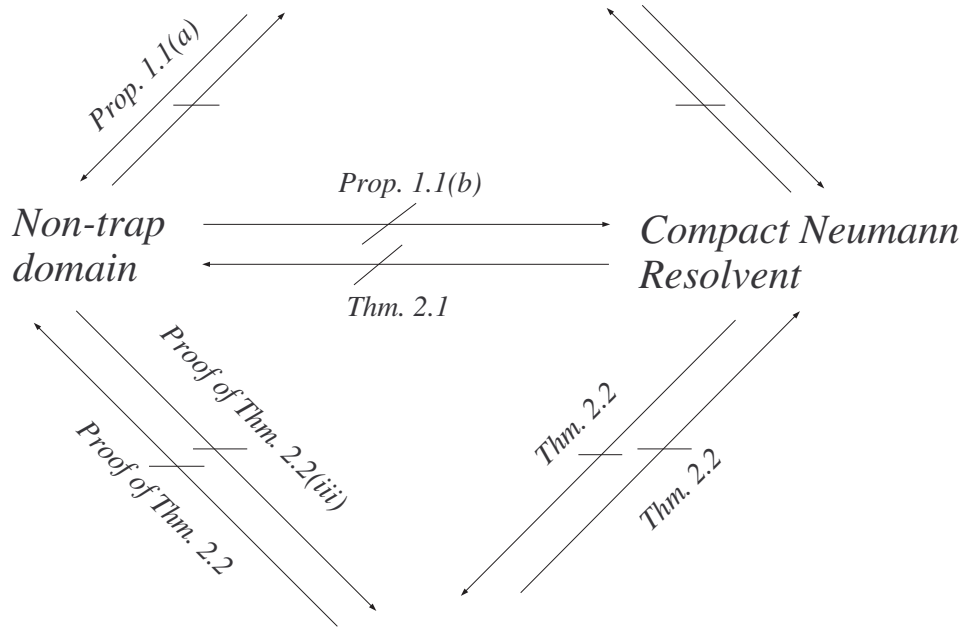

Intrinsic Ultracontractivity

FIGURE 1.

The following question was posed by Davies and Simon in [DS1, p. 372]: Is there a relationship between the compactness of the 1-resolvent of the Neumann Laplacian and intrinsic ultracontractivity of the Dirichlet Laplacian in a given domain? In Theorem 2.2 below, we will prove that there is no logical relationship between the two properties, i.e., all four logical combinations of the two properties and their negations occur in some domains. We will show in Remark 2.3 that there is no logical relationship between IU and being a non-trap domain. In summary, for a domain $D$ with finite volume, except for the obvious relation that the PHP implies $D$ is non-trap and the 1-resolvent of the Neumann Laplacian in $D$ is compact, there are no other logical relationships between the following properties: non-trap, IU, discrete spectrum of the Neumann Laplacian and PHP. The logical implications, or rather the lack of these, are depicted in Figure 1.

\section{THEOREMS AND PROOFS}

Suppose $f:[1, \infty) \rightarrow(0, \infty)$ is a Lipschitz function and let the corresponding horn domain $D_{f}$ be defined by

$$
D_{f}=\left\{(x, y) \in \mathbb{R}^{2}: x>1,|y|<f(x)\right\} .
$$

Theorem 2.1. (i) Suppose $D=D_{f} \subset \mathbb{R}^{2}$ is a horn domain. If $D$ is a non-trap domain, then the 1-resolvent of the Neumann Laplacian is compact.

(ii) There exists a trap domain $D$ where the 1-resolvent of the Neumann Laplacian is compact. Hence the Neumann Laplacian has a discrete spectrum in D but the parabolic Harnack principle does not hold.

Proof. By Proposition 2.11 of $[\mathrm{BCM}], D=D_{f}$ is a trap domain if and only if

$$
\int_{1}^{\infty}\left(\int_{1}^{x} \frac{1}{f(y)} d y\right) f(x) d x=\infty
$$


(i) Suppose $D=D_{f} \subset \mathbb{R}^{2}$ is not a trap domain. Then $\int_{1}^{\infty}\left(\int_{1}^{x} \frac{1}{f(y)} d y\right) f(x) d x$ $<\infty$. Hence

$$
\begin{aligned}
0 & =\lim _{T \rightarrow \infty} \int_{T}^{\infty}\left(\int_{1}^{x} \frac{1}{f(y)} d y\right) f(x) d x \geq \limsup _{T \rightarrow \infty} \int_{T}^{\infty}\left(\int_{1}^{T} \frac{1}{f(y)} d y\right) f(x) d x \\
& =\limsup _{T \rightarrow \infty}\left(\int_{T}^{\infty} f(x) d x\right)\left(\int_{1}^{T} \frac{1}{f(y)} d y\right) .
\end{aligned}
$$

Thus by a theorem of Evans and Harris (see [EH] or [DS2]), the 1-resolvent of the Neumann Laplacian in $D$ is compact.

(ii) Let $D_{f}$ be the horn domain with $f(x)=e^{-x^{2}}$. We have

SO

$$
\int_{1}^{x} \frac{1}{f(y)} d y=\int_{1}^{x} e^{y^{2}} d y \geq \frac{1}{x} \int_{1}^{x} y e^{y^{2}} d y=\frac{1}{2 x}\left(e^{x^{2}}-e\right)
$$

$$
\int_{1}^{\infty} \int_{1}^{x} \frac{1}{f(y)} d y f(x) d x \geq \int_{1}^{\infty} \frac{1}{2 x}\left(e^{x^{2}}-e\right) e^{-x^{2}} d x=\infty .
$$

This shows that $D_{f}$ is a trap domain. We also have

$$
\int_{1}^{x} \frac{1}{f(y)} d y=\int_{1}^{x} e^{y^{2}} d y \leq \int_{1}^{x} y e^{y^{2}} d y=\frac{1}{2}\left(e^{x^{2}}-e\right)
$$

and

so

$$
\int_{x}^{\infty} f(y) d y=\int_{x}^{\infty} e^{-y^{2}} d y \leq \frac{1}{x} \int_{x}^{\infty} y e^{-y^{2}} d y=\frac{1}{2 x} e^{-x^{2}}
$$

$$
\lim _{x \rightarrow \infty}\left(\int_{1}^{x} \frac{1}{f(y)} d y\right)\left(\int_{x}^{\infty} f(y) d y\right)=0 .
$$

In view of the results of Evans and Harris (see [EH], [DS2]), this implies that the 1-resolvent of the Neumann Laplacian in $D_{f}$ is compact.

It follows from Proposition 1.1(b) that the conclusion of Theorem 2.1(i) is not true for general planar domains with finite volume. We would find it interesting to know whether the conclusion of Theorem 2.1(i) is true for more general domains. Calculations similar to those in the proof for Theorem 2.1(ii) show that if one takes $f(x)=e^{-x^{\alpha}}$, then the resulting domain is a trap domain for $\alpha \leq 2$ and the 1-resolvent of the Neumann Laplacian is compact for $\alpha>1$.

Theorem 2.2. There exist domains $D_{k}, k=1,2,3,4$, having finite volumes with the following properties.

(i) $D_{1}$ is IU and it satisfies the parabolic Harnack principle (hence the 1-resolvent of the Neumann Laplacian on $D_{1}$ is compact).

(ii) $D_{2}$ is IU and the 1-resolvent of the Neumann Laplacian on $D_{2}$ is not compact.

(iii) $D_{3}$ is not $I U$ and it satisfies the parabolic Harnack principle (hence the 1-resolvent of the Neumann Laplacian on $D_{3}$ is compact).

(iv) $D_{4}$ is not IU and the 1-resolvent of the Neumann Laplacian on $D_{4}$ is not compact.

Proof. (i) It is easy to check that the unit disc is IU and it satisfies the parabolic Harnack principle.

(ii) and (iv) Let $D_{f} \subset \mathbb{R}^{2}$ be a horn domain with $f(x)=x^{\alpha}$ where $\alpha<0$. None of these domains has a compact 1-resolvent of the Neumann Laplacian, according 
to [EH] and [DS2]. The results of Bañuelos and Davis [BD] show that if $\alpha<-1$, then $D_{f}$ is IU but it is not IU if $-1 \leq \alpha<0$.

The above may suggest that the result depends on the finiteness of the volume of the domain. To show that this is not the case, we consider a multidimensional horn domain

$$
D_{f}=\left\{\left(x_{1}, x_{2}, \ldots, x_{d}\right) \in \mathbb{R}^{d}: x_{1}>1, \sqrt{x_{2}^{2}+\cdots+x_{d}^{2}}<f\left(x_{1}\right)\right\},
$$

which has finite volume for any $f(x)=x^{\alpha}$ with $\alpha<-1 / 2$ and $d \geq 3$. By the results of Evans and Harris [EH] (see also Davies and Simon [DS2]), $D_{f} \subset \mathbb{R}^{d}, d \geq 2$, does not have a compact 1-resolvent of the Neumann Laplacian for any $f(x)=x^{\alpha}$ with $\alpha<0$. If $\alpha<-1$, then $D_{f}$ is IU by Theorem 1.1(i)(A) of Bass and Burdzy BB1. If $-1<\alpha<0$, then $D_{f}$ is not IU, as indicated in Section 4 of [BB1].

(iii) We first recall a few well-known facts from the homogenization theory for the killed diffusions. Let $K \subset \overline{B(0,1 / 2)} \subset \mathbb{R}^{2}$ be the 1-dimensional classical Cantor set in the unit interval $[-1 / 2,1 / 2]$ sitting on the $x$-axis in the plane. Note that $K$, whose Hausdorff dimension is $\frac{\log 2}{\log 3}$, has positive logarithmic capacity and so it will be hit by planar Brownian motion. Let $K_{1}=\bigcup_{x \in \mathbb{Z}^{2}}(K+x)$, let $B_{t}$ be the Brownian motion in $\mathbb{R}^{2}$, and for $A \subset \mathbb{R}^{2}$, let $T_{A}^{B}=\inf \left\{t>0: B_{t} \in A\right\}$. It is standard to show (see Baxter, Chacon and Jain [BCJ]) that for any $\varepsilon>0$ there exists $r<\infty$ such that for $x \in B(0,2)$ we have $\mathbb{P}^{x}\left(T_{K_{1}}^{B}>T_{\partial B(0, r)}^{B}\right)<\varepsilon$. Let $K_{a}=a K_{1}$ for $a>0$. By scaling, $\mathbb{P}^{x}\left(T_{K_{a}}^{B}>T_{\partial B(0, a r)}^{B}\right)<\varepsilon$.

Let $x_{k}=\left(2^{-k}, 1 / 2\right), A_{k}=\left(B\left(0,2^{-k-2}\right) \backslash B\left(0,2^{-k-3}\right)\right)+x_{k}$, and $F=\bigcup_{k>1} A_{k} \cap$ $K_{b_{k}}$, where the $b_{k}$ 's will be chosen later in the proof. Set $U=(0,1)^{2}$ and $D=U \backslash F$. Since $K$ has zero 1-dimensional Hausdorff measure, so does $F$. Hence by Theorem 3.3 and Remark 2 in Chen [C], $F$ is a deletable set for the Sobolev space $W^{1,2}$; that is, $W^{1,2}(U \backslash F)=W^{1,2}(U)$. It follows that the 1-resolvent for the Neumann Laplacian on $D=U \backslash F$ is unchanged when $D=U \backslash F$ is replaced by the square $U$ and so it is compact.

Next we will show that $D$ is not IU. Let $Q_{k}=B\left(x_{k}, 2^{-k-3}\right)$ and let $p_{t}^{D}(x, y)$ and $p_{t}^{Q_{k}}(x, y)$ be the heat kernels in $D$ and $Q_{k}$, respectively, with the Dirichlet boundary conditions. A standard argument based on scaling and eigenfunction expansions shows that for some $0<c_{1}, \beta<\infty$ and all $k \geq 1$,

$$
\int_{Q_{k}} p_{1}^{D}\left(x_{k}, y\right) d y \geq \int_{Q_{k}} p_{1}^{Q_{k}}\left(x_{k}, y\right) d y \geq c_{1} \exp \left(-\beta 2^{-2 k}\right) .
$$

Fix some $\alpha>\beta$ and let $M=B((3 / 4,1 / 2), 1 / 16)$. Let $C_{k}=\partial B\left(x_{k}, 3 \cdot 2^{-k-4}\right)$ and, using the result from the first paragraph of the proof for (iii), choose $b_{k}>0$ so small that for some $c_{2}<\infty$, all $k \geq 1$ and every $x \in C_{k}$,

$$
\mathbb{P}^{x}\left(T_{K_{b_{k}}}^{B}>T_{\partial D}^{B}\right)<c_{2} \exp \left(-\alpha 2^{2 k}\right) .
$$

The probability that the Brownian motion starting from $x_{k}$ will hit $M$ before exiting $D$ is bounded from above by the probability that it will cross $A_{k}$ without hitting $K_{b_{k}}$. By the strong Markov property applied at the hitting time of $C_{k}$ and (2.3), this probability is bounded by $c_{2} \exp \left(-\alpha 2^{2 k}\right)$. It follows that the probability that the Brownian motion killed on the boundary of $D$ will be in $M$ at time $t=1$ is 
bounded by the same quantity. In other words,

$$
\int_{M} p_{1}^{D}\left(x_{k}, y\right) d y \leq c_{2} \exp \left(-\alpha 2^{2 k}\right)
$$

This and (2.2) imply that

$$
\lim _{k \rightarrow \infty} \frac{\int_{M} p_{1}^{D}\left(x_{k}, y\right) d y}{\int_{Q_{k}} p_{1}^{D}\left(x_{k}, y\right) d y}=0 .
$$

Let $x_{0}$ be the center of $M$. As $\left|Q_{k}\right| \rightarrow 0$,

$$
\lim _{k \rightarrow \infty} \frac{\int_{M} p_{1}^{D}\left(x_{0}, y\right) d y}{\int_{Q_{k}} p_{1}^{D}\left(x_{0}, y\right) d y}=\infty .
$$

We claim that this and (2.4) show that we cannot have

$$
\frac{p_{1}^{D}(x, y)}{p_{1}^{D}(x, z)} \geq c_{4} \frac{p_{1}^{D}(v, y)}{p_{1}^{D}(v, z)}
$$

for some $c_{4}$ and all $x, y, z, v \in D$. Suppose (2.6) is true, rewrite it in the product form, take $x=x_{k}$ and $v=x_{0}$, then integrate $y$ over $M$ first and integrate $z$ over $Q_{k}$ next, to obtain

$$
\frac{\int_{M} p_{1}^{D}(x, y) d y}{\int_{Q_{k}} p_{1}^{D}(x, z) d z} \geq c_{4} \frac{\int_{M} p_{1}^{D}(v, y) d y}{\int_{Q_{k}} p_{1}^{D}(v, z) d z}
$$

which contradicts the conjunction of (2.4) and (2.5).

The inequality (2.6) is a consequence of intrinsic ultracontractivity (see Davis D]) so $D$ is not IU. We note that according to Davis [D, the intrinsic ultracontractivity holds if and only if the condition (2.6) is satisfied for all $t>0$, not just for $t=1$ (where $c_{4}$ may depend on $t$ ).

Remark 2.3. (i) The proof of Theorem 2.2(iii) is based on the fact that one can delete a set $K$ of positive logarithmic or Newtonian capacity from $D$ but nevertheless the reflecting Brownian motion in $D \backslash K$ has the same law as that of the reflecting Brownian motion in $D$, or equivalently, $W^{1,2}(D \backslash K)=W^{1,2}(D)$ (see Chen [C]). Here we use sets $K_{a} \subset \mathbb{R}^{2}$ whose removal or addition does not affect the distribution of the reflected Brownian motion because they have zero 1-dimensional Hausdorff measure but are not negligible from the point of view of the killed Brownian motion because they have positive logarithmic capacity. We believe that using such special sets is not essential. Instead, one could use a countable number of densely packed slits in $A_{k}$, pointing towards $x_{k}$. This change would not affect in an essential way the proof of the lack of the IU property. The ideas and methods developed in Bass and Burdzy [BB2] in relation to "fiber Brownian motion" strongly suggest that the 1-resolvent for the Neumann Laplacian in this modified domain would be compact.

(ii) We have pointed out in part (ii) of the proof for Theorem 2.2 that according to Bañuelos and Davis [BD], any horn domain $D_{f}$ in $\mathbb{R}^{2}$ with $f(x)=x^{\alpha}$ and $\alpha<-1$ is IU, while, by (2.1), it is a trap domain. On the other hand, the domain $D$ in part (iii) of the proof for Theorem 2.2 is clearly a non-trap domain, since $W^{1,2}(D)=$ $W^{1,2}\left((0,1)^{2}\right)$ and so $D$ is a $W^{1,2}$-extension domain. We have shown that this domain is not IU. We conclude that there is no logical relationship between the IU property and trap domains, which is similar to the lack of relationship between the IU property and compactness of the 1-resolvent of the Neumann Laplacian. 


\section{ACKNOWLEDGMENTS}

We are grateful to Rodrigo Bañuelos, Brian Davies and Don Marshall for helpful comments.

\section{REFERENCES}

[BB] R. Bañuelos and K. Burdzy, On the "hot spots" conjecture of J. Rauch. J. Func. Anal. 164, (1999) 1-33. MR.1694534 (2000m:35085)

[BD] R. Bañuelos and B. Davis, A geometrical characterization of intrinsic ultracontractivity for planar domains with boundaries given by the graphs of functions. Indiana Univ. Math. J. 41, (1992) 885-913. MR1206335 (94g:60142)

[BB1] R. F. Bass and K. Burdzy, Lifetimes of conditioned diffusions. Probab. Theory Relat. Fields 91, (1992) 405-443. MR.1151804 (93e:60155)

[BB2] R. F. Bass and K. Burdzy, Fiber Brownian motion and the 'hot spots' problem. Duke Math. J. 105, (2000) 25-58. MR.1788041 (2001g:60190)

[BCJ] J. Baxter, R. Chacon and N. Jain, Weak limits of stopped diffusions. Trans. Amer. Math. Soc. 293, (1986) 767-792. MR.0816325 (87e:60125)

[BCM] K. Burdzy, Z.-Q. Chen and D. E. Marshall, Traps for reflected Brownian motion. Math. Z. 252 (2006) 103-132.

[C] Z.-Q. Chen, Reflecting Brownian motions and a deletion result for Sobolev spaces of order (1,2). Potential Analysis, 5 (1996), 383-401. MR1401073 (97f:46048)

[DS1] E. B. Davies and B. Simon, Ultracontractivity and the heat kernel for Schrödinger operators and Dirichlet Laplacians. J. Funct. Anal. 59, (1984) 335-395. MR0766493(86e:47054)

[DS2] E. B. Davies and B. Simon, Spectral properties of Neumann Laplacian of horns. Geom. Funct. Anal. 2, (1992) 105-117. MR 1143665 (93g:35099)

[D] B. Davis, Intrinsic ultracontractivity and the Dirichlet Laplacian. J. Func. Anal. 100, (1991) 162-180. MR1124297 (92k:35065)

[EH] W. D. Evans and D. J. Harris, Sobolev embeddings for generalized ridged domains. Proc. London Math. Soc. 54, (1987) 141-175. MR0872254 (88b:46056)

Department of Mathematics, Box 354350, University of Washington, Seattle, WashINGTON 98115-4350

E-mail address: burdzy@math.washington.edu

Department of Mathematics, Box 354350, University of Washington, Seattle, WashINGTON 98115-4350

E-mail address: zchen@math.washington.edu 